\title{
Towards context-free Semantic Localisation
}

\author{
Gabriele Marini \\ The University of Melbourne \\ marinig@student.unimelb.edu.au
}

\author{
Jorge Gonçalves \\ The University of Melbourne \\ jorge.goncalves@unimelb.edu.au
}

\author{
Eduardo Velloso \\ The University of Melbourne \\ eduardo.velloso@unimelb.edu.au
}

\author{
Raja Jurdak \\ Data61 - CSIRO \\ Raja.Jurdak@data61.csiro.au
}

\begin{abstract}
We propose a context-free semantic localisation approach to visualise and analyse indoor movements. We focus on settings where indoor location or rooms have strongly associated semantics, such as hospitals. We describe an approach that can work with different localisation systems, with little knowledge of the physical space properties, and with minimal bootstrapping required. We propose a movement representation that consists of time-encoded strings, and discuss how our approach can be used for analysing and visualising longitudinal indoor localisation data.
\end{abstract}

\section{CCS CONCEPTS}

- Human-centered computing $\rightarrow$ Empirical studies in $\mathrm{HCI}$ • Human-centered computing $\rightarrow$ Ubiquitous and mobile computing; • Human-centered computing $\rightarrow$ Smartphones.

\section{KEYWORDS}

Localisation analytics, indoor localisation

\section{ACM Reference Format:}

Gabriele Marini, Jorge Gonçalves, Eduardo Velloso, Raja Jurdak, and Vassilis Kostakos. 2019. Towards context-free Semantic Localisation. In Adjunct Proceedings of the 2019 ACM International foint Conference on Pervasive and Ubiquitous Computing and the 2019 International Symposium on Wearable Computers (UbiComp/ISWC '19 Adjunct), September 9-13, 2019, London, United Kingdom. ACM, New York, NY, USA, 8 pages. https://doi.org/10.1145/3341162.3349329

Permission to make digital or hard copies of all or part of this work for personal or classroom use is granted without fee provided that copies are not made or distributed for profit or commercial advantage and that copies bear this notice and the full citation on the first page. Copyrights for components of this work owned by others than the author(s) must be honored. Abstracting with credit is permitted. To copy otherwise, or republish, to post on servers or to redistribute to lists, requires prior specific permission and/or a fee. Request permissions from permissions@acm.org. UbiComp/ISWC '19 Adjunct, September 9-13, 2019, London, United Kingdom (c) 2019 Copyright held by the owner/author(s). Publication rights licensed to ACM.

ACM ISBN 978-1-4503-6869-8/19/09..\$15.00

https://doi.org/10.1145/3341162.3349329

\author{
Vassilis Kostakos \\ The University of Melbourne \\ vassilis.kostakos@unimelb.edu.au
}

\section{INTRODUCTION}

Understanding people's movements in relation to their environments is essential for both researchers and workplaces. Humans spend a big portion of their time in buildings where often each room or space has associated semantics in the context of people's flow. In Universities, for example, libraries, laboratories, and canteens have different meanings, and similarly hospitals have a reception, operating rooms, recovery rooms, etc. Traditionally, indoor localisation research has focused on improving the accuracy of localisation systems, developing a multitude of technologies that can accurately pin-point assets in space. Yet, independently of the actual localisation system used, indoor movement often happens between rooms or spaces on a map, and analysing longitudinal data in terms of those rooms is not straightforward. We currently lack a movement-centered representation that is flexible enough to work with a variety of localisation systems, and also allows researchers to study people's flow across indoor spaces and rooms.

In this paper we present such a room-centered approach to analyse and represent longitudinal indoor localisation data, by representing the rooms' semantics. Our goal is a technology-agnostic representation that abstracts movements from the physical space and its coordinates, and turns those into rooms and their semantics. Deploying a localisation system can often involve a long and tedious process which often requires on-site surveys and a training phase. Our aim is to build a context-free semantic localisation system which can work with different underlying localisation systems, which requires no training and a minimal knowledge of the physical space. Our approach is based on the idea of strongly typed spaces, where every room has a very specific role in people's or an organisation's flow. Therefore we expect our approach to be better-suited for environments where rooms can be represented as classes rather than coordinates. The motivation for our approach is to allow researchers to utilise data from an underlying localisation system, and focus more on the semantic visualisation and analysis of indoor spaces and people's flow between them.

We validate our approach on data collected at the Northern Health hospital in Melbourne. We deployed a Bluetooth Low 
Energy (BLE) indoor localisation system that uses proximity to detect the presence of people in rooms. Unconventionally, our system adopts a flipped scenario where beacons are carried by users and phones are anchored to walls. The collected RSSI values are first smoothed out, grouped and finally encoded to identify the presence of people in rooms, and subsequently their movement between rooms. We encode movements as a discrete time series of characters, and the resulting string effectively represents the time spent in each room. We measure the accuracy of our system by comparing the encoded sensor data with on-site collected ground truth using string similarity algorithms. Our results show that even with a simple proximity based localisation system, our approach can accurately detect and represent movement through rooms. We discuss how a string-based representation enable researchers to use existing string analysis algorithms used for pattern detection.

\section{RELATED WORK}

Indoor localisation[4] has been an active area of research for decades. Although there is currently no commercially technological standard for indoor localisation, many of the applications rely on radio signals and RSSI values[7]. Most of the research aims at either developing new Indoor Positioning Systems (IPS) or improving the existing ones' accuracy. Stemming from the system design and accuracy, there is a multitude of applications: People and asset tracking[3, 14], proximity-based services such as location-based advertisements or museum guides[18], indoor navigation both for people and robots[5]. Although these applications address a wide range of challenges, there are few systems that analyse and study the way people move around in indoor environments.

\section{Indoor Localisation and Mobility}

Yassin et al.[26] provide an extensive overview of the current available techniques. Most of the techniques use signals such as Infrared (IR) or Radio Frequency (RF) coupled with metrics such as: Time of Arrival (ToA), Time Difference of Arrival (TDoA), Received Signal Strength (RSS) and Angle of Arrival (AoA) to compute the location. These metrics are usually then analysed with techniques such as trilateration to determine the position in relation to other nodes.

One of the earliest projects on indoor localisation with sensor networks was by Want et al.[23], with the Active Badge where users carried a badge which emitted unique IR signals picked up by sensors deployed in predefined locations. Active Bat $^{1}$ [10] and Cricket Location Support System[17] applied ultrasound technology to substantially increase in the overall accuracy in measuring distance and inferring location.
With the proliferation of Radio Frequency (RF) transmitters, mobile computing devices and local-area wireless networks (LANs), the need for additional infrastructure was greatly overcome. Systems such as RADAR[1] and LANDMARC[16] relied on existing infrastructure and Wi-Fi Access Points to collect Received Signal Strength (RSS) values from RF- based devices carried by users. However, some systems focused more on indoor mobility. Hnat et al.[12] mounted ultrasonic range finders on doorways to detect people moving through them and infer their presence in the room.

In recent years Bluetooth became pervasively available on consumer devices, which can be used to locate people indoor through small Bluetooth tags placed throughout a building[27].

Bluetooth localisation is based on RSSI and techniques such as fingerprinting [20] or triangulation [22]. Most of the RSSI-based analysis use a path loss equation and RSSI values in time to estimate the distance [19]: $P L=P L_{d 0}+10 n \times$ $\log _{10}\left(\frac{d}{d_{0}}\right)+X_{\sigma}$ where $P L_{d 0}$ is the RSSI value at the distance $d_{0}$ used as reference. For simplicity, usually $d_{0}$ assumes the value of 1 , meaning that $P L_{d 0}$ would be the RSSI value at the distance of $1 \mathrm{~m}$ from the receiver. Finally, $d$ represents the actual distance from the receiver and $n$ is the path loss exponent which depends on the environment: Free space, indoor open-floor, or indoor with obstructions. Previous studies investigated the feasibility of using BLE beacons in indoor localisation [8], and found out that RSSI greatly varies according to the environment and to the amount of deployed beacons. Usually, more beacons correlates with an improved accuracy.

The recent Bluetooth Low Energy (BLE) subsystem and Apple's own implementation, iBeacon [15] helped reduce battery consumption and maintenance costs through small and inexpensive devices. Tests[9] show benefits in using BLE technology with an error $<2.6 \mathrm{~m}$ in $95 \%$ of measurements for a dense BLE network and up to an error of $<4.8 \mathrm{~m}$ for a more sparse deployment, an improvement WiFi based systems.

Unfortunately, conditions are not always ideal and different obstacles and materials make RSSI readings not reliable enough and need some further processing. The preprocessing step usually adopts the usage of smoothing algorithms. Deak et al.[6] compared smoothing algorithms for RSSI and localisation such as Fast Smoothing, Median Filtering, and Kalman Filtering. The 1-dimensional median filtering scored the lowest average error due to its ability to nullify small signal's variations.

Considering the performance of BLE and the low cost for BLE beacons, we adopted this technology in our localisation system. Additionally, due to our requirements of having minimal knowledge of the physical space, we decided to not 
rely on the path loss model. Instead, we used a median filtering on a proximity based RSSI system. Although beacons are usually mounted on the walls and user as anchor nodes for fingerprinting or trilateration, we use an inverted approach where people carry BLE beacons and active trackers are mounted on the walls.

\section{Spatial models}

Our approach is based on the notion of "strongly typed" rooms or locations that can be represented as a class instead of a set of coordinates. Once we obtain the RSSI values and infer the presence of a person in a room, we then represent this data in a semantic manner. In the literature, spaces are often represented as graphs and analysed through algorithms based on graph theory.

Becker et al.[2] summarise existing models for indoor spaces. Two classes of coordinates can be identified, geometric and symbolic. Geometric location models define positions in terms of coordinate tuples and geometric figures, and can provide information about the distance to be traveled to move across rooms. Geometric models often require an accurate modeling of the building's spaces.

On the other hand, proximity and fingerprinting based localisation systems provide information in terms of abstract symbols such as sensor identifiers (WiFi AP, BLE beacons or smartphones identifier). Symbolic location models abstract from the spatial properties and only take into account the relationships between spaces. Graph-based models are a type of symbolic model which use vertices as symbolic coordinates representing the rooms and edges to represent a connection between two rooms. Graph-based models are the only ones that support the definition of "connected to", making them suitable for modeling user movement. Graph models are often coupled with proximity analysis [11] to detect the presence of devices in rooms. Jensen et al.[13] modeled people's movements through a graph based model representing room relative locations and accessibility, and a graph of deployed nodes.

Analytics based on movements from indoor localisation are not new, but it is often based on occupancy data which can differ with different localisation systems. Wang et al.[21] propose a way to infer indoor location from occupancy data and note how occupancy data may not preserve privacy. Yaeli et al.[25] at IBM applied indoor localisation to understand customers behaviour by tracking shoppers and matching their data with POS data. Similarly, Yada [24] studied customer behaviour in stores through RFID technology, representing patterns as a character-encoded time series, parsed with the EBONSAI parsing system. The movement data is encoded with two attributes: a character representing the shop section, and a number representing the ratio of time spent by the customer in each section on the total time spent in the store.

For instance, a customer $x$ who spent 11 minutes in the store, visiting section $A$ for 2 minutes, then section $B$ for 3 minutes, section $\mathrm{C}$ for 4 minutes, and finally section $\mathrm{B}$ again for 2 minutes can be represented as $A B C B$, where each character encodes the ratio over the total time spent in the shop.

To the best of our knowledge, the literature focuses on either location accuracy or time spent in each room without considering the rooms and the building as a whole. In some cases such as Doorjamb, the room presence is inferred by non-tracking sensors and might not be applicable to environments with large or even absent doorways. Typically, we find that rooms are considered as a proxy for people's flow, and analyses tend to shift the focus on their connection and how people move between them. Little work has considered how people's movement captures, enacts, or deviates from operational processes or business processes that may actually drive people's movement. The work by Yada focuses on short trips and a single room, while we hope to apply a revision of this representation to a larger scale, sensing room-scale presences over long-term periods.

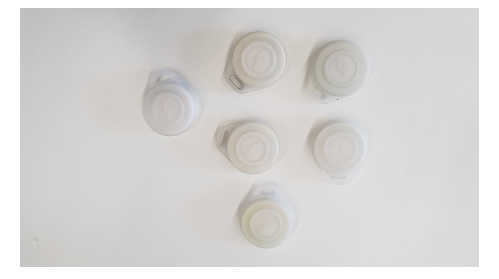

(a) Beacons

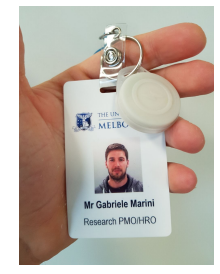

(b) Beacons size

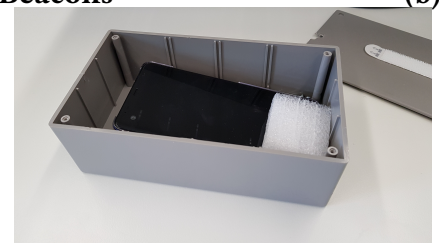

(c) Android phones

Figure 1: Android devices mounted to the walls, and beacons handed out to users.

\section{PROPOSED APPROACH}

We developed and deployed an indoor localisation system in a hospital setting to study the way staff (nurses, surgeons, doctors) and patients move throughout the building. The hospital setting provides a set of challenges which heavily impacted our design of the underlying localisation system:

- Nurses, surgeons or patients might not be carrying their phones at all times due to both privacy and hygiene concerns. This is challenging since most of the 
localisation systems rely on the deployment of beacons (or any kind of signal broadcasting device) as fixed anchor nodes, and smartphones as active trackers.

- Hospitals are critical environments where efficiency is essential. The carried tracking device should therefore be small, non-invasive and lightweight.

- Due to the nature of hospitals, deploying additional infrastructure would disrupt the hospital's workflow. WiFi-based localisation systems rely on the deployment of a dense network or WiFi Access Points.

After considering these issues and challenges we opted for an inverted BLE-based localisation system. In our system (Fig. 1) users such as nurses or patients carry a RadBeacon Dot, a BLE-based beacon that broadcasts its signal at configurable intervals and strength. We deployed Android phones as anchor nodes in each room of the floor and developed a custom Android app to continuously scan the surroundings for BLE beacons. The beacons use Apple's iBeacon standard.

\section{Preliminary tests}

The advertisement rate and transmission power of the beacons play an important role in the beacon's battery life. For this reason we ran a preliminary test to assess trade-off in terms of battery life and frequency of advertisements. We positioned 9 beacons with a combination of distance (at 1,2 and 5 meters from the tracker) and transmission rate (at 1,5 and $10 \mathrm{~Hz}$ ), and a transmission power of $-12 \mathrm{dBm}$. The results in Fig. 2 show how packets' inter-arrival time greatly increases at low advertisement rates even at close distance. On the other hand, a higher advertisement rate translates to shorter inter-arrival times also due to a shorter interval between possible missing packets. From this figure we identify a sweet-spot between 5 and $10 \mathrm{~Hz}$, which can achieve a constant stream of high frequency packets even at longer distances.

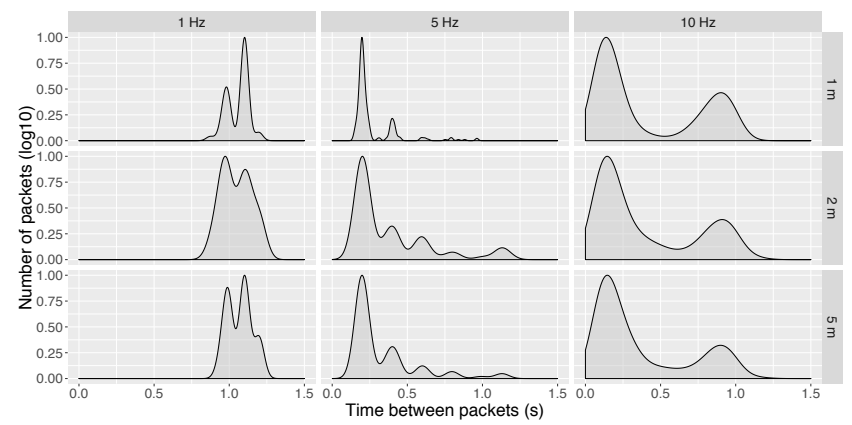

Figure 2: Advertisements per delta time (scaled)

We conducted a test at the hospital to see how the transmission power influences the maximum tracking distance. Results showed a high variance in the tracking distance, ranging between 3-6 meters with a transmission power of -9
$\mathrm{dBm}$. Finally, considering an average walking speed of 1.4 $\mathrm{m} / \mathrm{s}$, we opted for a transmission rate of $5 \mathrm{~Hz}$, to ensure that even in case of emergency a person wearing a beacon would not be missed by trackers in-range.

\section{EVALUATION}

We deployed a total of 16 phones in each room of the surgery ward (Fig. 3), with one per room except for larger rooms (with one side larger than 3 meters, which could potentially lose packets). We also deployed 20 beacons to 20 staff members who carried beacons for a total of 2 weeks. In addition we conducted our own independent validation of the system by having our researchers carry tags and walk throughout the ward while manually recording their trip details.

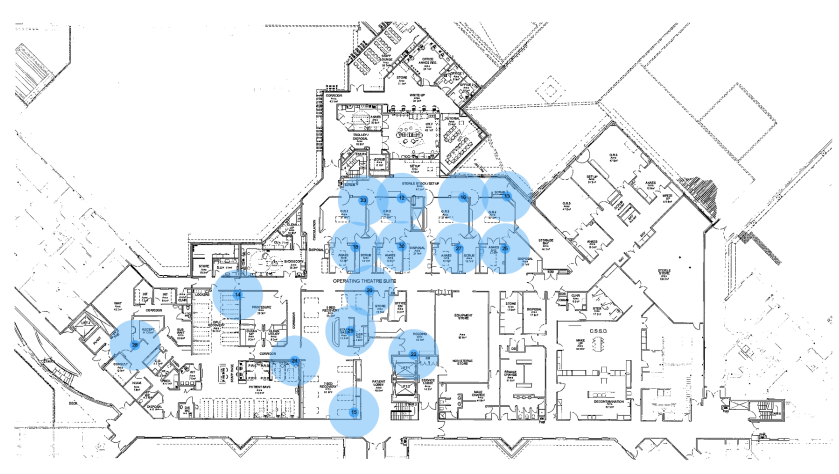

Figure 3: Hospital floor plan and deployed phones

The Android smartphones run a custom developed application that constantly scans the surrounding for beacons, capturing the follwing data and periodically uploading it to a server:

- Timestamp: The epoch time at which a beacon's packet was received.

- ID: The iBeacon minor and major are used to identify the beacon.

- RSSI

- UUID and IMEI of the phone that received the packet.

\section{Filtering}

We designed the localisation system to detect the presence of beacons in each room by using proximity with RSSI values over time. Drawing from the existing literature [6], tests showed the median filter as an accurate smoothing algorithm when compared to our collected ground truth Fig.4a.

The Median filter allows us to retain time-encoded information while discarding the noise caused by the Bluetooth signal fluctuations. In our deployment, we are more interested in correctly classifying the room-level position of the beacon, making sure it is correctly detected in the room it which it was at the time. Due to the limited space on the 


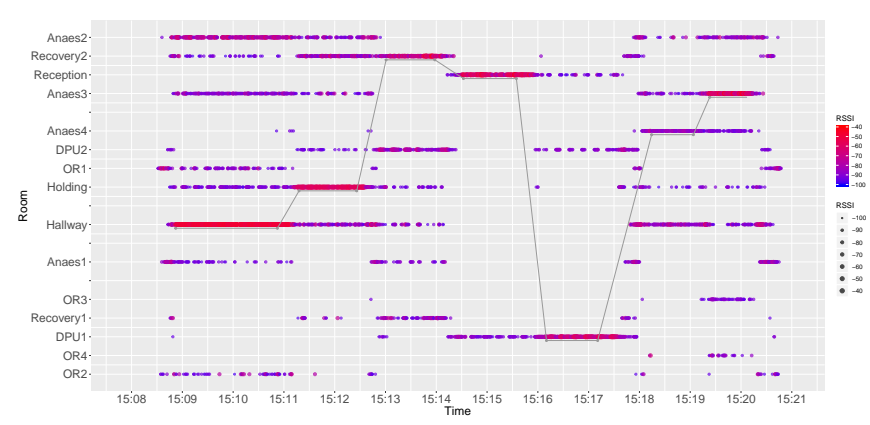

(a) Raw RSSI and ground truth (grey line)

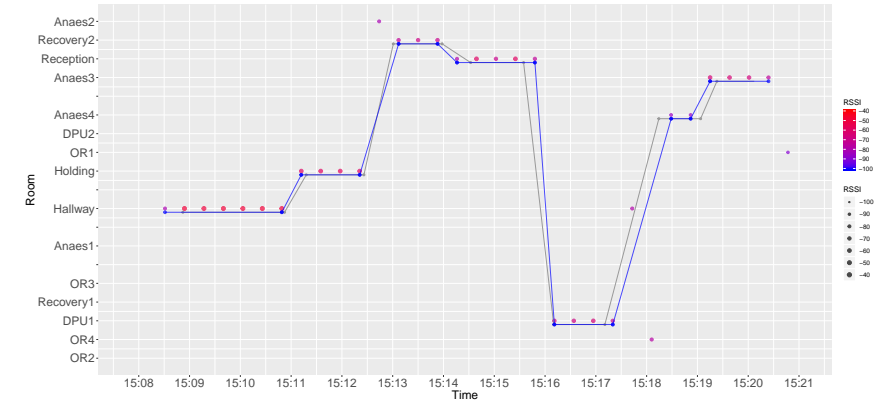

(b) Filtered RSSI (points) and reconstructed trip (in blue) Figure 4: Reconstructing the movement of a surgeon through the surgery ward (x-axis: time. $y$-axis: location)

rooms walls, it is often the case that two phones can be mounted on the shared wall in different rooms. From our tests, signals from beacons in one room can be picked up by phones mounted on the shared wall in an adjacent room, with a weaker RSSI. We smoothed the RSSI values through the median filter per room, to ensure rooms do not influence each other. Finally, we select the room where a beacon was most likely to be by selecting the maximum median RSSI in each time window across all rooms. In extreme cases where two or more rooms reported the maximum median RSSI in the same time frame, we relied on packet counting and selected the room which received the highest amount of packets in the time frame. The results in Fig. $4 \mathrm{~b}$ show the final smoothed signal and the reconstructed trip (blue line) compared with the collected ground truth (grey line).

To reconstruct people's movements we used a Run-Length Encoding (RLE) to join the resulting smoothed data points in a time-series. The maximum RSSI values from the previous step are connected with at least 2 consecutive values from the same room. For instance, if the maximum RSSI for a beacon $B$ was detected in room $R$ for a period of 10 minutes, the resulting RLE would return a single observation that contains the start and end times where $B$ was deemed to be in room $R$, and then presumably move to another room.

We empirically identified certain thresholds and heuristics to filter the resulting trips:

- Trips to the same room which are not separated by trips in different rooms can be merged into a single trip if the distance between the end of the first and the beginning of the second one is shorter than 5 minutes. This is due to the size of the hospital floor and the size of the rooms.

- If a beacon was nowhere to be tracked and there are no trips in any room in the last 5 minutes since the last trip, we consider the beacon as lost as no assumptions can be made about its location.

\section{Movement and pattern analysis}

We propose to represent the movement over time as a string of time-encoded characters so that it can be analysed by various string and pattern-matching techniques. Additionally, a string representation allows the use of Regular Expressions to define and detect patterns in the resulting string. For instance, consider the trip shown in Table 1 . We can represent this trip as a string in which each letter defines the presence of the beacon in a $\tau$ time window. In this example, if the time window is of size $w=5$ minutes, we can rewrite the movements as a string:

\section{ААВВВВВВССССССССАAАAАA} $D D D D D D B B B B$

\section{Performance}

Our results show that the reconstructed trips from the raw RSSI data closely match the collected ground truth. We quantify errors as the measure of distance between the ground truth and our result, using string similarity algorithms such as Levenshtein and Optimal String Alignment. The error is computed as the ratio between the string distance and the total length of the string. The results shown in Fig. 5 show the effect of the median time window size (used during filtering) over the RSSI readings. The disparity between the ground truth (as collected by researcher walking around the hospital) and the estimated trip (as inferred by our system) increases as the time window increases. This suggests that

Table 1: String representation of a trip. Each character represents a period of 5 minutes.

\begin{tabular}{cccccc}
\hline Room & Ch. & Start & End & Dur. & String \\
\hline Preparation & A & 0 & 10 & 10 & AA \\
Anaesthesia & B & 10 & 40 & 30 & BBBBBB \\
Operating Room & C & 40 & 80 & 40 & CCCCCCCC \\
Preparation & A & 80 & 110 & 30 & AAAAAA \\
Unknown & - & 110 & 130 & 20 & \\
Recovery & D & 130 & 160 & 30 & DDDDDD \\
Anaesthesia & B & 160 & 180 & 20 & BBBB \\
\hline
\end{tabular}


the granularity level of the collected ground truth plays an important role in the measurement error. Furthermore, a larger time window would not be able to represent room stays shorter than the time window itself.

In our observations we notice that patients tend to spend a larger portion of their time in a single room while nurses regularly move between rooms. Given this range of behaviours, we observe that a window size of 24 seconds provides the best results with our collected ground truth. Fig. 6 shows how the error changes as the time window for each character in the string increases. A larger time window per character in the string representation also impacts the string length. If trips are shorter than the time window, we only report the first room during that period. The choice of the string time window has a lower impact compared to the median time window.

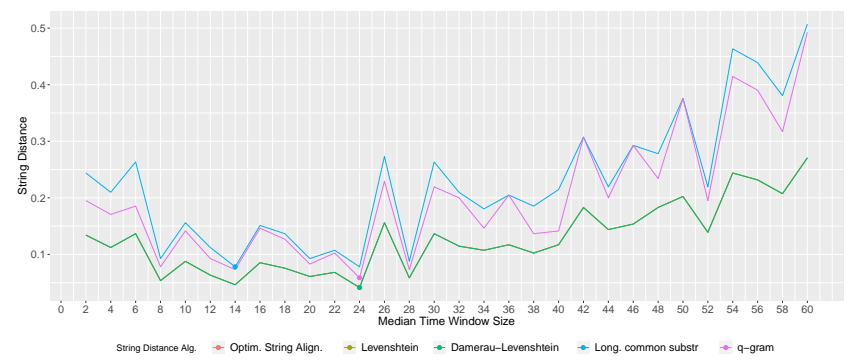

Figure 5: Effect of the median time window (used during filtering) on observation errors. As the time window increases, the error (distance from the ground truth) increases.

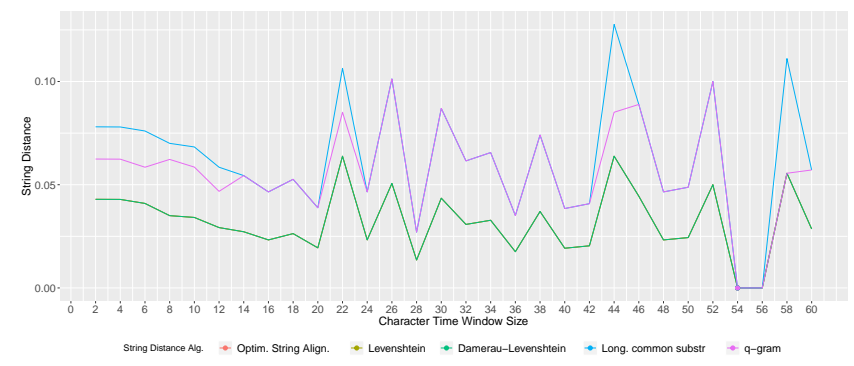

Figure 6: Effect of the character time window on the distance from the ground truth. Longer time windows tend to result in bigger error.

\section{DISCUSSION AND CONCLUSION}

The flexibility of our proposed approach can be highlighted if we consider ways in which the collected data can help organizations understand and analyze people's movements inside bulidings. For example, we refer back to the trip shown in Table 1. Given the string that represents this trip, we can define a regular expression to detect patterns. For instance if we define the following pattern: $/ C\{5\} A^{+}{ }_{-}{ }^{*} D / g$ we can detect a pattern of $5 \mathrm{Cs}$ in the string followed by at least one $A$, some or no empty characters, and ending with a $D$. By running this regular expression over our string we can then identify the presence of the pattern:

\section{$A A B В B В B В С С C С C C C C A A A A A A \_$DDDDDDBBBB}

Considering the window size $w$, the pattern we were looking for identifies the following amount of time spent in each room:

- 25 minutes in the Operating Room (C), followed by

- at least 5 minutes in the Preparation room (A), and

- 5 minutes in the Recovery room (D).

Our example shows how representing movement as a string enables us to use string processing algorithms and techniques - such as Regular Expressions - to define and even query trips in the whole data-set of reconstructed movements. Effectively, this allow us to describe the processes in a building, and identify instances that match or deviate from a process. For example, in our discussions with hospital administrators and surgeons, we have come to understand that a hospital expects that after a patient is anaesthetized they move immediately into the operating room. Hence, we can search and identify those segments of traces where a patient spent more than 5 minutes in the anaesthesia room but did not subsequently go to the operating room. That would be one example of operating room "down-time", and hospital managers who seek to improve operations would love to have statistics and information on how often this happens, for which patients, over long periods of time. In our case, once we identify all instances matching this pattern, we would be able to provide aggregate summaries of when, why and how the down-time occurred, and present them to hospital administration for taking action and setting policies. Furthermore, having access to real-world logs from hospitals would allow us to further improve our system accuracy, comparing our operating room down-time results with the data collected manually by the hospital.

Beyond the use of Regular Expressions, it is interesting to note that a Context-Free Grammar may be a useful tool to analyse this data. While regular expressions can detect the presence on a pattern, it is hard to identify deviations from expected patterns. Using a context-free grammar, we can define business processes as a set of rules, and then searching through the string-encoded trips we can identify where and when we have deviations from the expected patterns. This is akin to a parser complaining about a syntax error in a source code: it is possible to identify which rule is broken and at which point in the text. This presents an additional direction that we are considering for our future work. It should be noted that a Context-Free Localisation is not directly related to the use Context-Free grammar: A context-free localisation 
system aims at detecting movements without training or without site-specific parameters, and a context-free grammar is one of the ways to achieve that.

In this paper we propose a way to analyse RSSI proximity data to detect indoor movements across rooms. Additionally, we propose a string-based representation for capturing and analysing indoor movement. We deployed a BLE localisation system in an hospital which uses an inverted design where people cannot carry their smartphones at all times, which are instead mounted on the rooms walls. Using RSSI proximity and a median filter we can then localise the carried beacons in each room. Our representation can be used with other indoor localisation systems as it works on a middle layer of abstraction and it is not based on coordinates. We compared the reconstructed movements with our collected ground truth by using string similarity algorithms. Our results show that this representation can be accurate and can be used together with existing string and pattern analysis algorithms to study indoor mobility and flow.

In the future we will aim to develop tools for further analysing longitudinal indoor mobility datasets, querying those datasets, and visualising them. We also aim to use our string-based representation with other underlying localisation technologies such as WiFi. Finally, we plan on comparing our representation with existing graph-based models, and study the feasibility of using this model for pattern detection and machine learning algorithms.

\section{ACKNOWLEDGMENTS}

Gabriele Marini is supported by a $\mathrm{PhD}$ scholarship provided by Data61-CSIRO. Eduardo Velloso is the recipient of an Australian Research Council Discovery Early Career Award (grant DE180100315) funded by the Australian Government. This work is partially funded by a Samsung Global Research Outreach grant, and the ARC Discovery Project DP190102627.

\section{REFERENCES}

[1] P. Bahl and V. N. Padmanabhan. 2000. RADAR: an in-building RF-based user location and tracking system. In Proceedings IEEE INFOCOM 2000 Conference on Computer Communications. Nineteenth Annual foint Conference of the IEEE Computer and Communications Societies (Cat. No.00CH37064), Vol. 2. IEEE, 775-784 vol.2. https://doi.org/10.1109/ INFCOM.2000.832252

[2] Christian Becker and Frank Dürr. 2005. On location models for ubiquitous computing. Personal and Ubiquitous Computing 9, 1 (01 Jan 2005), 20-31. https://doi.org/10.1007/s00779-004-0270-2

[3] Igor Bisio, Andrea Sciarrone, and Sandro Zappatore. 2016. A new asset tracking architecture integrating RFID, Bluetooth Low Energy tags and ad hoc smartphone applications. Pervasive and Mobile Computing 31 (2016), 79 - 93. https://doi.org/10.1016/j.pmcj.2016.01.002

[4] Krishna Chintalapudi, Anand Padmanabha Iyer, and Venkata N. Padmanabhan. 2010. Indoor Localization Without the Pain. In Proceedings of the Sixteenth Annual International Conference on Mobile Computing and Networking (MobiCom '10). ACM, New York, NY, USA, 173-184. https://doi.org/10.1145/1859995.1860016

[5] S. Chumkamon, P. Tuvaphanthaphiphat, and P. Keeratiwintakorn. 2008. A Blind Navigation System Using RFID for Indoor Environments. In 2008 5th International Conference on Electrical Engineering/Electronics, Computer, Telecommunications and Information Technology, Vol. 2. 765768. https://doi.org/10.1109/ECTICON.2008.4600543

[6] Gabriel Deak, Kevin Curran, and Joan Condell. 2010. Evaluation of Smoothing Algorithms for a RSSI-Based Device-Free Passive Localisation. In Image Processing and Communications Challenges 2, Ryszard S. Choraś (Ed.). Springer Berlin Heidelberg, Berlin, Heidelberg, 469-476.

[7] Gabriel Deak, Kevin Curran, and Joan Condell. 2012. A survey of active and passive indoor localisation systems. Computer Communications 35, 16 (2012), 1939 - 1954. https://doi.org/10.1016/j.comcom.2012.06.004

[8] Ramsey Faragher and Robert Harle. 2014. An analysis of the accuracy of bluetooth low energy for indoor positioning applications. In Proceedings of the 27th International Technical Meeting of the Satellite Division of the Institute of Navigation (ION GNSS+âÁZ14). 201-210.

[9] R. Faragher and R. Harle. 2015. Location Fingerprinting With Bluetooth Low Energy Beacons. IEEE Journal on Selected Areas in Communications 33, 11 (Nov 2015), 2418-2428. https://doi.org/10.1109/JSAC.2015. 2430281

[10] Andy Harter, Andy Hopper, Pete Steggles, Andy Ward, and Paul Webster. 2002. The anatomy of a context-aware application. Wireless Networks 8, 2/3 (2002), 187-197.

[11] J. Hightower and G. Borriello. 2001. Location systems for ubiquitous computing. Computer 34, 8 (Aug 2001), 57-66. https://doi.org/10. 1109/2.940014

[12] Timothy W. Hnat, Erin Griffiths, Ray Dawson, and Kamin Whitehouse. 2012. Doorjamb: Unobtrusive Room-level Tracking of People in Homes Using Doorway Sensors. In Proceedings of the 10th ACM Conference on Embedded Network Sensor Systems (SenSys '12). ACM, New York, NY, USA, 309-322. https://doi.org/10.1145/2426656.2426687

[13] C. S. Jensen, H. Lu, and B. Yang. 2009. Graph Model Based Indoor Tracking. In 2009 Tenth International Conference on Mobile Data Management: Systems, Services and Middleware. 122-131. https://doi.org/ 10.1109/MDM.2009.23

[14] J. Koch, J. Wettach, E. Bloch, and K. Berns. 2007. Indoor Localisation of Humans, Objects, and mobile Robots with RFID Infrastructure. In 7th International Conference on Hybrid Intelligent Systems (HIS 2007). 271-276. https://doi.org/10.1109/HIS.2007.25

[15] X. Lin, T. Ho, C. Fang, Z. Yen, B. Yang, and F. Lai. 2015. A mobile indoor positioning system based on iBeacon technology. In 2015 37th Annual International Conference of the IEEE Engineering in Medicine and Biology Society (EMBC). IEEE, 4970-4973. https://doi.org/10.1109/ EMBC.2015.7319507

[16] L. M. Ni, Yunhao Liu, Yiu Cho Lau, and A. P. Patil. 2003. LANDMARC: indoor location sensing using active RFID. In Proceedings of the First IEEE International Conference on Pervasive Computing and Communications, 2003. (PerCom 2003). IEEE, 407-415. https: //doi.org/10.1109/PERCOM.2003.1192765

[17] Nissanka B. Priyantha, Anit Chakraborty, and Hari Balakrishnan. 2000. The Cricket Location-support System. In Proceedings of the 6th Annual International Conference on Mobile Computing and Networking (MobiCom '00). ACM, New York, NY, USA, 32-43. https: //doi.org/10.1145/345910.345917

[18] Omer Rashid, Paul Coulton, and Reuben Edwards. 2008. Providing Location Based Information/Advertising for Existing Mobile Phone Users. Personal Ubiquitous Comput. 12, 1 (Jan. 2008), 3-10. https: //doi.org/10.1007/s00779-006-0121-4 
[19] Jenny RÃúbesaat, Peilin Zhang, Mohamed Abdelaal, and Oliver Theel. 2017. An Improved BLE Indoor Localization with Kalman-Based Fusion: An Experimental Study. Sensors 17, 5 (2017). https://doi.org/10 3390/s17050951

[20] F. Subhan, H. Hasbullah, A. Rozyyev, and S. T. Bakhsh. 2011. Indoor positioning in Bluetooth networks using fingerprinting and lateration approach. In 2011 International Conference on Information Science and Applications. 1-9. https://doi.org/10.1109/ICISA.2011.5772436

[21] Xiao Wang and Patrick Tague. 2014. Non-Invasive User Tracking via Passive Sensing: Privacy Risks of Time-Series Occupancy Measurement. In Proceedings of the 2014 Workshop on Artificial Intelligent and Security Workshop (AISec '14). ACM, New York, NY, USA, 113-124. https://doi.org/10.1145/2666652.2666655

[22] Y. Wang, Xu Yang, Yutian Zhao, Yue Liu, and L. Cuthbert. 2013. Bluetooth positioning using RSSI and triangulation methods. In 2013 IEEE 10th Consumer Communications and Networking Conference (CCNC). IEEE, 837-842. https://doi.org/10.1109/CCNC.2013.6488558

[23] Roy Want, Andy Hopper, Veronica Falcão, and Jonathan Gibbons. 1992. The Active Badge Location System. ACM Trans. Inf. Syst. 10, 1 Jan.
1992), 91-102. https://doi.org/10.1145/128756.128759

[24] Katsutoshi Yada. 2011. String Analysis Technique for Shopping Path in a Supermarket. F. Intell. Inf. Syst. 36, 3 (June 2011), 385-402. https: //doi.org/10.1007/s10844-009-0113-8

[25] A. Yaeli, P. Bak, G. Feigenblat, S. Nadler, H. Roitman, G. Saadoun, H. J. Ship, D. Cohen, O. Fuchs, S. Ofek-Koifman, and T. Sandbank. 2014. Understanding Customer Behavior Using Indoor Location Analysis and Visualization. IBM 7. Res. Dev. 58, 5-6 (Sept. 2014), 3:1-3:12. https: //doi.org/10.1147/JRD.2014.2337552

[26] A. Yassin, Y. Nasser, M. Awad, A. Al-Dubai, R. Liu, C. Yuen, R. Raulefs, and E. Aboutanios. 2017. Recent Advances in Indoor Localization: A Survey on Theoretical Approaches and Applications. IEEE Communications Surveys Tutorials 19, 2 (Secondquarter 2017), 1327-1346. https://doi.org/10.1109/COMST.2016.2632427

[27] L. Zhang, X. Liu, J. Song, C. Gurrin, and Z. Zhu. 2013. A Comprehensive Study of Bluetooth Fingerprinting-Based Algorithms for Localization. In 2013 27th International Conference on Advanced Information Networking and Applications Workshops. 300-305. https: //doi.org/10.1109/WAINA.2013.205 